\title{
Interação Mãe-Criança e Problemas de Comportamento Infantil em Crianças com Hipotireoidismo Congênito ${ }^{1}$
}

\author{
Carla Krás Borges Figueiredo \\ Pontifícia Universidade Católica do Rio Grande do Sul \\ Ligia Schermann \\ Universidade Luterana do Brasil
}

\begin{abstract}
Resumo
O presente estudo tem como objetivo averiguar se há diferenças na interação mãe-criança e nos problemas de comportamento infantil em dez crianças com hipotireoidismo congênito (HC), tratadas precocemente, comparadas com um grupo de dez crianças sadias. Quando as crianças estavam com idades entre quatro e seis anos as interações foram filmadas durante uma situação de jogo livre e pontuadas, conforme protocolo de observação Play Observation Scheme and Emotional Rating (POSER). As mães responderam ao Child Behavior Checklist 4/18 (CBCL). Não houve diferença significativa, tanto na interação, quanto nos problemas de comportamento entre os dois grupos. A ausência de diferença significativa entre os grupos mostra a importância do tratamento precoce em crianças com HC, o que facilita a qualidade da interação mãe-criança e ameniza problemas de comportamento infantil.
\end{abstract}

Palavras-chave: Interação mãe-criança; problemas de comportamento infantil; hipotireoidismo congênito.

Mother-Infant Interaction and Behavior Problems in Child With Congenital Hypothyroidism

\begin{abstract}
The present study intended to find out whether there are differences in mother-child interaction as well as to detect childhood behavior problems in ten children suffering from congenital hypothyroidism (HC), who had received early treatment, in comparison with a group of ten healthy children. When the children were between four-six years of age the interaction was videotaped during a free play situation and was assessed according to the Play Observational Scheme and Emotion Rating (POSER). The mothers answered the Child Behavior Checklist 4/18 (CBCL). No significant differences were found in motherinfant interaction measures, or on the child behavior problems. The lack of significant difference between the groups attests for the importance of early treatment in children with $\mathrm{HC}$, which facilitates the quality mother-infant interaction and soften behavior problems in children.

Keywords: Mother-infant interaction; child behavior problems; congenital hypothyroidism.
\end{abstract}

Nas últimas décadas, inúmeros estudos, entre eles os de Goldberg e DiVitto (1983), Capuzzi (1989), Barnard (1997), têm mostrado a importância da interação mãecriança no posterior desenvolvimento social, emocional e cognitivo da criança. Bowlby $(1976,1988)$ evidenciou os primeiros meses de vida da criança como primordiais para o desenvolvimento da conduta de apego entre o bebê e sua mãe. $\mathrm{O}$ apego da mãe com a criança passou a ser entendido como resultante da ativação de sistemas comportamentais da mãe e da criança, cujo desenvolvimento independe de reforçadores externos como calor e comida. Neste sentido, o apego é tido como

1 Endereço para correspondência: Av. Francisco Trein, 416/211, Porto Alegre, CEP 91350-100, Fone: (51) 341-9383/98159853, E-mail: ckrasborges@uol.com.br / ligiasch@conex.com.br

${ }^{2}$ Este artigo é parte da Dissertação de Mestrado da primeira autora defendida no programa de Pós-Graduação em Psicologia da PUC-RS com bolsa do $\mathrm{CNPq}$, orientada pela segunda autora.

${ }^{3}$ Agradecimentos: as autoras agradecem ao Dr. Edmundo Kreisner pelo encaminhamento das crianças com hipotireoidismo congênito. um conjunto de comportamentos, passível de observação e descrição e evidencia o estabelecimento de uma relação afetiva e discriminativa com uma pessoa. Para Bowlby é um processo ativo, que pressupõe interação.

Um dos aspectos importantes da observação da interação mãe-criança é a sensitividade materna, conceito desenvolvido por Ainsworth, Blehar, Waters e Wall (1978) e definido como a habilidade da mãe em perceber, interpretar e responder de forma adequada e contingente aos sinais da criança. A mãe muito sensitiva é bastante atenta aos sinais da criança e responde a eles pronta e apropriadamente. É a mãe que consegue ver os acontecimentos do ponto de vista do bebê e que conhece qual o significado das comunicações dele, por mais sutis que elas sejam. No outro extremo, está a mãe muito insensitiva que parece agir quase exclusivamente de acordo com seus desejos, humores e atividades. A mãe pode responder aos sinais do bebê, mas o faz com atraso, e isso já é, em si, insensitivo. A sensitividade materna, portanto, assume uma posição central nas experiências 
interativas da criança pequena, contribuindo para sua futura organização comportamental e cognitiva (Barnard, 1997).

Além da importância do papel da mãe no processo da interação com a criança, estudos recentes evidenciam que, desde que nasce, o bebê tem o aparato sensorial e a capacidade de percepção bastante desenvolvidos, os quais são estimulados geralmente pela mãe ou cuidador primário. Assim, além de ter capacidades para colocaremse na interação com outras pessoas, as crianças, desde muito cedo, produzem uma série de respostas que determinam essa interação e o processo interativo passa a ser visto como recíproco e bidirecional (Brazelton, 1981; Bremner, 1988; Klaus \& Kennell, 1993). Wendland-Carro e Piccinini (1995) encontraram na revisão da literatura sobre a interação precoce mãe-bebê, algumas características do bebê que implicam diretamente no padrão interativo precoce do par mãe-bebê. Dentre estas características, encontra-se, por exemplo, a ordem do nascimento, o sexo do bebê, seu temperamento, sua responsividade para estímulos táteis, visuais e auditivos, sua aparência física, tais como tônus muscular e a postura, e seus estados de vigília.

A importância da interação mãe-criança para o desenvolvimento social da infância é tão marcante que Stern (1997) faz referência ao processo interativo como determinante do sintoma ou problema que traz uma família para tratamento. As queixas sobre a criança, geralmente relacionadas ao sono, alimentação, conduta ou apego, ou direcionadas à mãe, aparecem principalmente através da interação, pois é aí que os pais vivenciam estas queixas. Isto não é o mesmo que dizer que a causa de todos os problemas está na interação e, sim, que é através dela que surgem os sintomas primários.

Levando em conta as características de uma criança saudável, o processo interativo pode diferenciar-se quando a criança apresenta alguma doença crônica. A doença em uma pessoa da família é considerada uma situação de crise que pode ser mais ou menos difícil, dependendo da severidade da doença e de como ela é vivenciada. Quando quem adoece é uma criança, o modo como ela reage e a interação com o cuidador vai ser fundamental para o seu bem estar psicológico. Portanto, as reações da criança à doença dependem, não somente do seu nível de desenvolvimento cognitivo e emocional, da capacidade adaptativa prévia, do grau de sofrimento e mutilação, como também do significado que a doença tem para a criança e para seus pais, da relação pais-filho e da resposta da criança frente à reação dos pais (Lewis \& Volkmar, 1993).
Existem autores que enfatizam que a doença crônica em uma criança necessariamente desencadeará mais problemas de comportamento à essa criança. Os problemas mais importantes estão relacionados à dificuldade de relacionamento com outras pessoas, pois a criança pode vir a ser mais retraída devido ao seu estado de saúde (Lavigne \& Farer-Routman, 1992; Weiland, Pless \& Roghmann, 1992).

Por outro lado, há autores que preconizam a multifatoriedade para o desencadeamento de problemas comportamentais e dificuldades interacionais entre pais e filhos, quando o filho(a) apresenta alguma malformação ou doença crônica. Em uma reavaliação sobre o conceito de risco, Horowitz (1992) conclui que os estudos que abordam o status médico, juntamente com problemas no desenvolvimento e comportamento infantil, devam ser analisados juntamente com o contexto ambiental e as diferenças individuais.

Nolan e Pless (1986) realizaram um levantamento sobre crianças com risco médico e doenças crônicas e chamam a atenção para a complexidade deste tipo de estudo, uma vez que as conseqüências de uma malformação e de uma doença crônica são influenciadas por diversos fatores relacionados à criança, à doença em si, à família, ao contexto social em que os sujeitos estão inseridos e à situação médica da criança. Assim, a combinação de várias situações de risco como a severidade da doença e o contexto social, por exemplo, podem revelar um perigo maior para o desenvolvimento de problemas emocionais e comportamentais nestas crianças.

Goldberg e DiVitto (1983) observam que diferenças na interação mãe-filho desaparecem até o final do primeiro ano de vida da criança, quando comparadas díades em que a criança tem risco médico e díades em que este risco é ausente. No entanto, a mudança interacional vai depender do desenvolvimento da criança e do investimento familiar nos cuidados fornecidos a ela. Reafirmando este achado, Capuzzi (1989) mostra que as mães de crianças doentes apresentam maior dificuldade de interagir e apegar-se, quando as crianças completam um mês de idade, do que as mães de crianças saudáveis. No entanto, esta diferença não acontece quando o par mãe-criança é estudado aos seis e 12 meses de idade da criança. Esta melhora nos padrões de apego e sintonia da interação se dá quando o apoio social a estas mães é positivo.

Dentre as doenças que atingem a infância, o hipotireoidismo congênito é considerado uma malformação que pode ser diagnosticada bem cedo, logo que um bebê nasce, através do exame de sangue conhecido como "teste do pezinho". Ao ser constatada esta doença, a criança passa por diversos procedimentos de controle e reposição hormonal que diferem da rotina de uma 
criança saudável e, conseqüentemente, da rotina dos pais de uma criança saudável. Estes procedimentos acabam fazendo parte da vida destes indivíduos e de suas famílias. O hipotireoidismo congênito (HC) é uma doença endócrino-metabólica considerada como a principal causa para o aparecimento de uma deficiência mental, ocorrendo em uma freqüência de um em cada 4000 a 4500 recém-nascidos no exterior (La Franchi, 1982). No Brasil a incidência desta doença é de um em cada 2730 nascimentos (Neto \& Schulte, 1998). Na maioria dos casos, o HC é decorrente de uma anormalidade no desenvolvimento da glândula tireóide (hipotireoidismo primário) que tem como função, em condições normais, regular a temperatura corporal e a função cardiovascular, bem como atuar na maturação do sistema nervoso central. A ação dos hormônios da tireóide sobre o sistema nervoso central ocorre durante os últimos meses de gestação e os dois primeiros anos de vida (Mavaux, 1981). O prognóstico mental destas crianças depende principalmente da idade do início do tratamento de reposição hormonal e adequação do mesmo, associado à importância da natureza e da severidade do defeito congênito (Grant, Smith, Fuggle, Tokar \& Chapple, 1992).

Considerando que o HC é uma malformação congênita, tida como uma doença crônica pela necessidade de reparação hormonal para o resto da vida da criança, torna-se necessário entender melhor como se dá a influência dos aspectos físicos/médicos com o relacionamento familiar destas crianças, especialmente a interação com a mãe. O presente estudo examina a interação mãe-criança e os problemas de comportamento infantil em crianças com hipotireoidismo congênito em comparação com um grupo de crianças sadias.

\section{Método}

\section{Participantes}

Participaram do presente estudo 20 crianças, na faixa etária de quatro anos a seis anos e seis meses, e suas mães, divididas em dois grupos. O primeiro grupo constituído por dez crianças com diagnóstico de HC encaminhadas à Faculdade de Psicologia da Pontifícia Universidade Católica do Rio Grande do Sul para avaliação do desenvolvimento cognitivo e neuropsicomotor. Estas crianças fazem parte de um estudo mais amplo realizado em colaboração entre os cursos de Pós Graduação em Endocrinologia da Universidade Federal do Rio Grande do Sul e PósGraduação em Psicologia da PUCRS. As crianças foram encaminhadas por um médico endocrinologista, através de sua clínica particular, e do ambulatório de Endocrinologia Pediátrica do Hospital Materno-Infantil Presidente Vargas. Estas crianças representam uma amostra de crianças com HC do estado do Rio Grande do Sul, sendo que todas foram tratadas precocemente, antes dos três meses de idade (média de idade de início do tratamento 37 dias, desvio padrão de 22 dias).

Tabela 1. Descrição da Amostra: Variáveis Demográficas

\begin{tabular}{|c|c|c|c|c|c|c|}
\hline & $\begin{array}{l}\text { Idade } \\
(\mathrm{a}, \mathrm{m})\end{array}$ & $\begin{array}{c}\text { Crianças } \\
\text { Sexo }\end{array}$ & $\begin{array}{l}\text { Função } \\
\text { Cognitiva }\end{array}$ & $\begin{array}{c}\text { Mães } \\
\text { Escolaridade }\end{array}$ & $\begin{array}{c}\text { Pais } \\
\text { Escolaridade }\end{array}$ & $\begin{array}{l}\text { Família } \\
\text { Renda } \\
\text { (sm) }\end{array}$ \\
\hline $\mathrm{HC}$ & 4,8 & F & $\mathrm{M}$ & $3^{\circ}$ incom & $2^{\circ} \mathrm{compl}$ & 4 \\
\hline $\mathrm{C}$ & 4,7 & F & MS & $2^{\circ} \mathrm{compl}$ & $3^{\circ}$ incom & +4 \\
\hline $\mathrm{HC}$ & 6 & M & MI & $1^{\circ} \mathrm{compl}$ & $2^{\circ}$ incom & 2 \\
\hline $\mathrm{C}$ & 5,8 & M & M & $2^{\circ} \mathrm{compl}$ & $2^{\circ}$ incom & 3 \\
\hline $\mathrm{HC}$ & 6 & $\mathrm{~F}$ & M & $2^{\circ} \mathrm{compl}$ & $2^{\circ} \mathrm{compl}$ & +4 \\
\hline $\mathrm{C}$ & 6 & F & M & $2^{\circ} \mathrm{compl}$ & $1^{\circ} \mathrm{compl}$ & 4 \\
\hline $\mathrm{HC}$ & 6,5 & M & MI & $1^{\circ} \mathrm{compl}$ & $1^{\circ} \mathrm{compl}$ & +4 \\
\hline $\mathrm{C}$ & 6 & M & M & $2^{\circ}$ incom & $1^{\circ} \mathrm{compl}$ & +4 \\
\hline $\mathrm{HC}$ & 6,2 & M & S & $2^{\circ} \mathrm{compl}$ & $2^{\circ} \mathrm{compl}$ & +4 \\
\hline C & 5,5 & M & MS & $3^{\circ}$ incom & $2^{\circ} \mathrm{compl}$ & +4 \\
\hline $\mathrm{HC}$ & 4 & F & $\mathrm{M}$ & $1^{\circ} \mathrm{compl}$ & $1^{\circ} \mathrm{compl}$ & 4 \\
\hline C & 4 & F & MS & $2^{\circ}$ incom & $2^{\circ} \mathrm{compl}$ & 4 \\
\hline $\mathrm{HC}$ & 4 & M & MS & $3^{\circ} \mathrm{compl}$ & $3^{\circ} \mathrm{compl}$ & 4 \\
\hline C & 4 & M & S & $3^{\circ} \mathrm{compl}$ & $3^{\circ}$ incom & 4 \\
\hline $\mathrm{HC}$ & 5,7 & F & MI & $3^{\circ}$ incom & $3^{\circ} \mathrm{compl}$ & +4 \\
\hline $\mathrm{C}$ & 5,5 & F & M & $3^{\circ} \mathrm{compl}$ & $3^{\circ} \mathrm{compl}$ & +4 \\
\hline $\mathrm{HC}$ & 6 & F & M & $2^{\circ}$ incom & $3^{\circ} \mathrm{compl}$ & +4 \\
\hline $\mathrm{C}$ & 6,2 & F & M & $3^{\circ}$ incom & $3^{\circ}$ incom & +4 \\
\hline $\mathrm{HC}$ & 4 & M & M & $2^{\circ} \mathrm{compl}$ & $2^{\circ} \mathrm{compl}$ & +4 \\
\hline $\mathrm{C}$ & 4,1 & M & M & $2^{\circ} \mathrm{compl}$ & $2^{\circ}$ incom & +4 \\
\hline
\end{tabular}

HC: hipotireoidismo congênito C: controle S: superior; MS: médio superior; M: médio; MI: médio inferior sm: salários mínimos 
O segundo grupo (grupo controle) foi composto por dez crianças sem HC ou outra doença crônica. As crianças deste grupo foram selecionadas de três creches particulares e públicas de Porto Alegre e do interior do Estado, escolhidas por conveniência e foram emparelhadas com as crianças do primeiro grupo (com HC) conforme idade, sexo e posição da criança na constelação familiar. Procurou-se ainda aproximar a idade e nível de escolaridade da mãe, nível de escolaridade do pai e renda familiar, por considerar-se que estas variáveis poderiam interferir nas variáveis dependentes.

Em ambos os grupos, optou-se pela inclusão de crianças sem déficit cognitivo, avaliadas através das escalas Wechsler (WPPSI). Todas as mães do estudo possuem situação conjugal estável (casadas ou com união marital estável por mais de três anos). Os critérios de exclusão envolveram mães com história de enfermidade psiquiátrica, crianças que apresentaram função cognitiva baixa (limítrofes e deficientes mentais), mães adolescentes ou com mais de 45 anos e mães analfabetas.

A descrição da amostra encontra-se na Tabela 1.

\section{Observação Direta}

Optou-se pelo estudo da interação através de observação direta, tendo como base um protocolo de observação, Play Observation Scheme and Emotion Rating (POSER), desenvolvido por Wolke (1986). Este protocolo avalia itens referentes ao comportamento da mãe (quantidade de verbalizações, controle verbal do comportamento, sensitividade, expressão emocional positiva, expressão emocional negativa), ao comportamento da criança (quantidade de verbalizações, orientação da tarefa, atividade, intensidade, persistência/atenção, tom emocional) e itens referentes ao comportamento da díade (reciprocidade, controle da sessão, atmosfera geral). Cada um dos itens do protocolo é avaliado em uma escala de cinco pontos (Anexo A), considerando as pontuações mais elevadas condizentes a comportamentos interativos mais positivos.

Para o estudo dos problemas de comportamento, foi utilizado o Child Behavior Checklist 4/18 (CBCL) desenvolvido por Achenbach (1991). Foi aplicada a segunda parte do instrumento referente aos problemas de comportamento que podem estar presentes em crianças e adolescentes. O perfil comportamental é constituído de nove escalas individuais: I retraimento, II queixas somáticas, III ansiedade/depressão, IV problemas com o contato social, $\mathrm{V}$ problemas com o pensamento, VI problemas com a atenção, VII comportamento delinqüente, Vlll comportamento agressivo, IX problemas sexuais. Quando consideradas em conjunto, as escalas I, II e III são chamadas de Escalas de Introversão, já as escalas VII e VIII são chamadas Escalas de Extroversão. A soma dos escores obtidos nestas escalas corresponde ao total de problemas do comportamento (Achenbach, 1991; Bordin, Mari \& Caeiro, 1995).

\section{Questionário Sobre os Dados Demográficos}

Foi aplicado um questionário à mãe para a obtenção de informações sobre fatores sócio-econômicos e constelação familiar.

\section{Procedimento para a Coleta dos Dados}

A seleção das crianças com HC foi realizada a partir de um estudo mais amplo, levando em conta os critérios de inclusão e exclusão apresentados anteriormente. Após informação prestada à mãe sobre o estudo que se pretendia realizar e a assinatura de um termo de concordância quanto à participação de seu filho(a), todas as crianças foram avaliadas quanto ao nível cognitivo, através das escalas Wechsler (WPPSI), e as mães responderam ao questionário sobre os dados demográficos. As crianças com HC foram encaminhadas ao Serviço de Atendimento Psicológico da PUCRS para avaliação e as crianças do grupo controle foram avaliadas em suas respectivas creches.

Todas as crianças foram filmadas em vídeo com suas mães durante cinco minutos, em uma situação de jogo livre, com brinquedos não estruturados como lego e cubos. Após a filmagem da interação, a mãe respondeu ao CBCL. Optou-se pela aplicação deste instrumento por um entrevistador como forma de padronizar o entendimento das mães nas questões a serem respondidas.

Antes do início da análise dos filmes, foi realizado um estudo piloto com seis crianças que não participaram da presente pesquisa. $\mathrm{O}$ estudo piloto possibilitou a adequação dos instrumentos utilizados para coleta dos dados, bem como a realização de um treinamento do protocolo de observação e teste de acordo entre observadores. $\mathrm{O}$ acordo foi obtido através da pontuação de dois observadores independentes que examinaram as categorias de Wolke (1986) durante as sessões de filmagem das díades deste estudo piloto. A percentagem de acordo obtida oscilou entre $67 \%$ a $100 \%(m=82,5 ; d p=12)$, sendo calculada pelo número de acordos dividido pelo número de acordos mais o número de desacordos.

\section{Resultados}

\section{Observação da Interação Mãe-Criança}

Os resultados mostram que houve uma dispersão na pontuação do protocolo de observação. Comparandose os escores um e dois e quatro e cinco, nos itens maternos 
observa-se uma maior prevalência de pontuação entre os escores quatro e cinco do que entre os escores um e dois ( $45 \%$ e $5 \%$, respectivamente), indicando muitas verbalizações por parte da mãe, baixo controle verbal do comportamento da criança $(60 \%$ e $15 \%)$ e mais sensitividade $(25 \%$ e $20 \%)$. Não houve, no entanto, manifestação de expressão emocional positiva e nem negativa (Tabela 2).

Tabela 2. Percentagem de Pontuação dos Itens Maternos

\begin{tabular}{lllllll}
\hline & \multicolumn{7}{c}{$\%$} \\
\hline Itens Maternos & & 1 & 2 & 3 & 4 & 5 \\
\hline Quantidade de & HC & 0 & 10 & 50 & 40 & 0 \\
verbalizações & $\mathrm{C}$ & 0 & 0 & 50 & 50 & 0 \\
Controle verbal & $\mathrm{HC}$ & 0 & 0 & 40 & 40 & 20 \\
do comportamento & $\mathrm{C}$ & 0 & 30 & 10 & 40 & 20 \\
Controle e & $\mathrm{HC}$ & 10 & 20 & 40 & 20 & 10 \\
tutoramento & $\mathrm{C}$ & 0 & 20 & 30 & 20 & 30 \\
Sensitividade & $\mathrm{HC}$ & 0 & 20 & 70 & 10 & 0 \\
& $\mathrm{C}$ & 0 & 20 & 40 & 40 & 0 \\
Expressão emocional & $\mathrm{HC}$ & 70 & 10 & 20 & 0 & 0 \\
positiva & $\mathrm{C}$ & 0 & 50 & 30 & 20 & 0 \\
Expressão emocional & $\mathrm{HC}$ & 100 & 0 & 0 & 0 & 0 \\
negativa & $\mathrm{C}$ & 100 & 0 & 0 & 0 & 0 \\
\hline
\end{tabular}

Nos itens referentes ao comportamento da criança durante a interação aparece uma dispersão maior entre os escores quatro e cinco e um e dois, indicando que a maioria das crianças verbalizou muito $(50 \%$ e $15 \%$, respectivamente) e estavam orientadas para a tarefa $(35 \%$ e $5 \%$ ), permanecendo menos ativa $(0 \%$ e $90 \%)$ e interagindo com menos intensidade ( $0 \%$ e $80 \%)$. A persistência e a atenção na tarefa foi mais baixa do que alta e o tom emocional era de felicidade moderada (Tabela 3).

Tabela 3. Percentagem de Pontuação dos Itens da Criança

\begin{tabular}{lllllll}
\hline & \multicolumn{7}{c}{$\%$} \\
\hline Itens da Criança & & 1 & 2 & 3 & 4 & 5 \\
\hline Quantidade de & HC & 0 & 30 & 10 & 50 & 10 \\
verbalizações & $\mathrm{C}$ & 0 & 0 & 60 & 30 & 10 \\
Orientação para & HC & 0 & 10 & 50 & 40 & 0 \\
a tarefa & $\mathrm{C}$ & 0 & 0 & 70 & 30 & 0 \\
Atividade & HC & 0 & 80 & 20 & 0 & 0 \\
& $\mathrm{C}$ & 0 & 100 & 0 & 0 & 0 \\
Intensidade & HC & 70 & 10 & 20 & 0 & 0 \\
& $\mathrm{C}$ & 60 & 20 & 20 & 0 & 0 \\
Persistência/Atenção & HC & 10 & 40 & 40 & 10 & 0 \\
& $\mathrm{C}$ & 0 & 50 & 30 & 20 & 0 \\
Tom emocional & HC & 0 & 0 & 80 & 20 & 0 \\
& $\mathrm{C}$ & 0 & 0 & 70 & 20 & 10 \\
\hline
\end{tabular}

Nos itens da díade, através da comparação dos escores quatro e cinco e um e dois, observa-se que existe moderada e muita reciprocidade (50\% e $10 \%$, respectivamente). No que se refere ao controle da sessão, as pontuações indicam que ora a criança está no controle e a mãe acata e vice-versa, ora nenhuma das duas controla a sessão $(55 \%$ e $20 \%)$. A atmosfera geral na maioria das vezes foi neutra $(20 \%$ e $0 \%)$ conforme mostra a Tabela 4.

Tabela 4. Percentagem de Pontuação dos Itens da Díade

\begin{tabular}{lllllll}
\hline & \multicolumn{7}{c}{$\%$} \\
\hline Itens da Díade & & 1 & 2 & 3 & 4 & 5 \\
\hline Reciprocidade & HC & 0 & 10 & 50 & 30 & 10 \\
& C & 0 & 10 & 30 & 30 & 30 \\
Controle da sessão & HC & 0 & 30 & 0 & 50 & 20 \\
Atmosfera geral & C & 0 & 10 & 50 & 30 & 10 \\
& HC & 0 & 0 & 80 & 10 & 10 \\
& C & 0 & 0 & 80 & 0 & 20 \\
\hline
\end{tabular}

Para comparar a freqüência de cada categoria entre os grupos, utilizou-se o teste não paramétrico de Wilcoxon para amostras pareadas (Tabela 5). A análise estatística dos dados não mostrou diferença significativa entre os grupos em nenhum dos itens maternos. No entanto, observa-se uma tendência das mães das crianças com HC terem maior controle e tutoramento da sessão bem como manifestarem mais expressão emocional positiva do que o grupo controle.

Tabela 5. Teste de Wilcoxon para os Itens da Interação Mãe-Criança

\begin{tabular}{lll}
\hline Variáveis & $Z$ & $p$ \\
\hline Itens maternos & & \\
Quantidade de verbalizações & 0,707 & $0,49 \mathrm{~ns}$ \\
Controle verbal do comportamento & 0,531 & $0,60 \mathrm{~ns}$ \\
Controle e tutoramento & 1,613 & $0,11 \mathrm{~ns}$ \\
Sensitividade & 1,000 & $0,32 \mathrm{~ns}$ \\
Expressão emocional positiva & 1,633 & $0,10 \mathrm{~ns}$ \\
Expressão emocional negativa & 0,000 & $1,00 \mathrm{~ns}$ \\
Itens da criança & & \\
Quantidade de verbalizações & 0,289 & $0,77 \mathrm{~ns}$ \\
Orientação para a tarefa & 0,000 & $1,00 \mathrm{~ns}$ \\
Atividade & 1,414 & $0,15 \mathrm{~ns}$ \\
Intensidade & 0,973 & $0,33 \mathrm{~ns}$ \\
Persistência/Atenção & 0,632 & $0,53 \mathrm{~ns}$ \\
Tom emocional & 0,816 & $0,41 \mathrm{~ns}$ \\
Itens da díade & & \\
Reciprocidade & 0,513 & $0,60 \mathrm{~ns}$ \\
Controle da sessão & 0,513 & $0,60 \mathrm{~ns}$ \\
Atmosfera geral & 0,272 & $0,79 \mathrm{~ns}$ \\
\hline
\end{tabular}




\section{Problemas de Comportamento Infantil}

Os resultados do teste não paramétrico de Wilcoxon para amostras pareadas mostram que não houve diferença significativa entre os grupos estudados para cada escala do perfil comportamental do CBCL (Tabela 6).

Tabela 6. Teste de Wilcoxon para os Escores T do CBCL

\begin{tabular}{lll}
\hline Escalas & $Z$ & $p$ \\
\hline Retraimento & 0,210 & $0,83 \mathrm{~ns}$ \\
Queixas somáticas & 0,169 & $0,86 \mathrm{~ns}$ \\
Ansiedade/Depressão & 0,892 & $0,37 \mathrm{~ns}$ \\
Ansitmas com o contato social & 0,593 & $0,55 \mathrm{~ns}$ \\
Problemas com o pensamento & 0,420 & $0,67 \mathrm{~ns}$ \\
Problemas com a atenção & 0,833 & $0,40 \mathrm{~ns}$ \\
Comportamento delinqüente & 1,262 & $0,20 \mathrm{~ns}$ \\
Comportamento agressivo & 0,510 & $0,61 \mathrm{~ns}$ \\
Problemas com a sexualidade & 0,276 & $0,78 \mathrm{~ns}$ \\
\hline
\end{tabular}

A ausência de diferença significativa entre os grupos foi igualmente constatada para as escalas de introversão $(Z=0,63)$ e extroversão $(Z=1,33)$ do CBCL.

\section{Discussão}

A presente pesquisa investigou a interação mãe-criança e os problemas de comportamento infantil em crianças com hipotireoidismo congênito em idade pré-escolar, de quatro a seis anos, comparadas com um grupo de crianças sadias da mesma idade. Todas as crianças com HC iniciaram tratamento de reposição hormonal antes dos três meses de idade, o que caracteriza tratamento precoce para o hipotireoidismo.

Os resultados mais salientes foram a inexistência de diferenças significativas de comportamentos interativos mãe criança e de problemas comportamentais nos dois grupos estudados. Esses achados nos fazem pensar que nem sempre uma malformação/doença crônica em uma criança pequena precisa, necessariamente, dificultar o processo interativo entre a mãe e a criança, nem tampouco acarretar problemas de comportamento nessas crianças. Além disso, é importante abordar quais condições contribuíram para os padrões interativos mais positivos entre a mãe e a criança e a não observância, por parte da mãe, de problemas de comportamento em seus(suas) filhos(as). A literatura nos mostra a existência de fatores que minimizam o impacto de uma malformação/doença crônica em uma criança, entre eles, o apoio social e o atendimento médico adequado (Capuzzi, 1989; DeMaso e cols., 1991; Goldberg \& DiVitto, 1983).

Conforme observado anteriormente, os pais da amostra pertencem a uma população com educação média e com renda familiar de mais de quatro salários mínimos na maior parte das famílias. Estas características, certamente devem ter minimizado possíveis riscos sociais dos sujeitos envolvidos no presente estudo e que poderiam interferir nos resultados referentes à interação e aos problemas de comportamento. Alguns estudos mostram a importância dos fatores econômicos e de escolaridade dos pais no entendimento e na condução da doença de uma criança (Barnard, 1997), bem como o risco social que uma criança pode estar submetida quando inserida em um contexto familiar muito carente economicamente (Horowitz, 1992; Kopp, 1990).

Torna-se evidente, através dos resultados da presente pesquisa, a ausência da relação de causa e efeito entre uma malformação/doença crônica de uma criança e a interferência negativa no relacionamento com a mãe e no desencadeamento de problemas de comportamento. Os estudos de Nolan e Pless (1986), Kopp (1990) e Horowitz (1992) chamam a atenção para outras demandas e outros fatores de risco, além de somente a doença tais como, a condição social da família, a gravidade da doença e a qualidade relacional entre pais e filhos(as).

Estes achados levam a resgatar estudos que investigam a condição física de crianças pequenas e que demonstram a importância de se discutir a vulnerabilidade a que certas crianças estão submetidas. Neste sentido, cita-se a vulnerabilidade emocional, física e ambiental (Goldberg \& DiVitto, 1983; Horowitz, 1992; Kopp, 1990). Mesmo os estudos que procuram mensurar especialmente os processos pelos quais os comportamentos interativos entre mãe e criança ocorrem, o aspecto qualitativo da interação e a ação das variáveis ambientais são levados em conta (Goldberg, Gotowiec \& Simmons, 1995; Schermann, Hagekull, Bohlin, Person \& Sedin, 1997). No que se refere à vulnerabilidade física, as crianças com HC do presente estudo apresentam uma malformação interna da glândula tireóide (HC primário) responsável pelo bom funcionamento do sistema nervoso central - uma de suas principais funções (Mavaux, 1981). O fato de estas crianças terem sido tratadas precocemente, antes dos três meses de idade, certamente assegurou-lhes um bom prognóstico mental, com nível cognitivo preservado. Isto nos faz pensar que estas crianças estão resguardadas de repercussões adversas que o HC pode causar, a principal delas sendo a deficiência mental. Provavelmente, a invulnerabilidade física pode estar associada à invulnerabilidade emocional e social. Supõe-se que as famílias do estudo tiveram condições psicológicas e econômicas para investir no tratamento de seus filhos e que tiveram acesso ao atendimento médico especializado. 
O que o presente estudo apresenta é a interação mãecriança com mais aspectos positivos do que negativos encontrados nos dois grupos estudados. Este achado mostra que a condição de ser doente parece não afetar, de modo geral, a interação mãe-criança do grupo com HC. DeMaso e colaboradores (1991) atentam para a questão de que a percepção materna sobre a doença do(a) filho(a) tem influências no ajustamento da criança. Deste modo a doença assume o papel coadjuvante num espetáculo da vida que poderia ser trágico. O modo com que os pais reagem e a qualidade da interação, estes sim merecem o prêmio de facilitadores da adaptação e bem estar de crianças com malformação/doença crônica.

Pode-se observar na interação que as mães dos dois grupos estão graduadas como mais sensitivas do que insensitivas. Este dado demonstra que as mães da presente pesquisa estão sensíveis e responsivas aos sinais e comunicações de seus filhos(as).

Assim, as crianças com HC do presente estudo estão bem em relação à interação com a mãe a aos problemas de comportamento infantil. O estigma de ser uma criança com doença crônica parece não estar presente, pois a maioria está inserida socialmente na pré-escola e apresenta interação positiva com a mãe. O padrão interativo deve repercutir no dia-a-dia da díade e nas futuras socializações da criança. Do mesmo modo, as mães não consideram que seus filhos tenham sérios problemas de comportamento ao responderem ao CBCL.

Além disso, passados alguns anos do diagnóstico, e com o tratamento em andamento, os resultados da observação da interação indicam que as reações psicológicas mais intensas, especialmente da mãe, podem não interferir na interação com o(a) filho(a), pois o impacto da malformação já é bem menor. Associado ao atendimento adequado, no caso do HC através de programas de screening eficientes, pode-se supor que o fato das crianças deste estudo serem atendidas pelo mesmo médico desde o início do tratamento tenha reforçado o vínculo com o mesmo, facilitando a adesão ao tratamento e o processo de informação e conscientização da doença. Para Klaus e Kennell (1993), é importante que os pais saibam do que se trata a malformação e suas conseqüências, para amenizar fantasias que podem adquirir uma dimensão maior do que a realidade.

Diferindo do estudo de Goldberg e colaboradores (1995) que mostra crianças com comprometimento médico serem mais introvertidas, as crianças com HC do presente estudo demonstram não terem problemas com introversão comparadas com o grupo de crianças sadias. Entretanto, pode-se pensar que as crianças da Psicologia: Reflexão e Crítica, 2001, 14(3), pp. 487-495 presente pesquisa ainda são pequenas e que os problemas de introversão são menos perceptíveis à mãe neste momento, podendo aparecer mais tarde na idade escolar.

Considerando que o HC é uma malformação dentro do corpo e, portanto, o impacto de visualizá-la não acontece, como no caso de uma fissura lábio-palatal e hidrocefalia, por exemplo, talvez não produza um desconforto tão grande para a criança e para os pais da presente pesquisa. Estes fatores tomados em conjunto parecem ter minimizado o impacto causado pelo nascimento de uma criança com $\mathrm{HC}$ da presente amostra.

Através desse estudo pode-se concluir que o fato das crianças com HC terem sido tratadas precocemente, sempre pelo mesmo médico, estabelecendo boa adesão ao tratamento e serem provenientes de famílias com nível sócio-econômico médio, possa ter servido como fator preventivo para o não aparecimento de distúrbios no desenvolvimento social e comportamental dessas crianças na idade pré-escolar.

\section{Referências}

Achenbach, T. M. (1991). Manual for the Child Behavior Checklist/4-18 and 1991 Profile. Burlington, University of Vermont.

Ainsworth, M., Blehar, M., Waters, E. \& Wall, S. (1978). Patterns of attachment. Hillsdale, NJ: Erlbaum.

Barnard, K. E. (1997). Influencing parent-child interaction for children at risk. Em M. J. Guralnick (Org.), The effectiveness of early intervention (pp. 249-267). Baltimore: Paul H. Brookes.

Bordin, I. A. S., Mari, J. J. \& Caeiro, M. F. (1995). Validação da versão brasileira do "Child Behavior Checklist (CBCL)" (Inventário de Comportamentos da Infância e Adolescência): dados preliminares. Revista ABP-APAL, 17(2), 55-66.

Bowlby, J. (1976). El vinculo afectivo. Buenos Aires: Paidos.

Bowlby, J. (1988). Cuidados maternos e saúde mental. São Paulo: Martins Fontes.

Brazelton, T. B. (1981). Bebês e mamães. Rio de Janeiro: Campus.

Bremner, J. G. (1988). Infancy. Massachusetts: Basil Blackwell.

Capuzzi, C. (1989). Maternal attachment to handicapped infants and the relationship to social support. Research in Nursing and Health, 12, 161167.

DeMaso, D., Campis, L., Wypij, D., Bertram, S., Lipshitz, M. \& Freed, M. (1991). The impact of maternal perception and medical severity on the adjustment of children with congenital heart disease. Journal of Pediatric Psychology, 16(2), 137-149.

Goldberg, S. \& DiVitto, B. (1983). Born too soon - Preterm birth and early development. San Francisco: W.H. Freeman.

Goldberg, S., Gotowiec, A. \& Simmons, R. (1995). Infant- mother attachment and behavior problems in health and chronically ill preschoolers. Development and Psychopathology, 7, 267-282.

Grant, D. B., Smith, I., Fuggle, P. W., Tokar, S. \& Chapple, J. (1992). Congenital hypothyroidism detected by neonatal screening: relationship between biochemical severity and early clinical features. Archives Disease in Childhood, 67, 87-90.

Horowitz, F. D. (1992). The concept of risk: a reevaluation. Em S. L. Friedman \& M. D. Sigman (Orgs.), The psychological development of lowbirthweight children (pp. 61-88). Norwood: Ablex.

Klaus, M. H. \& Kennell, J. H. (1993). Pais / bebê - a formação do apego. Porto Alegre: Artes Médicas. 
Kopp, C. (1990). Risks in infancy: Appraising the research. Merrill-Palmer Quarterly, 36(1), 117-140.

La Franchi, S. L. (1982). Hypothyroidism congenital and acquired. Em S. A. Kaplan (Org.), Clinical pediatric and adolescent endocrinology (pp. 83-91) Philadelphia, Saunders.

Lavigne, J. \& Farer-Routman, J. (1992). Psychological adjustment to pediatric physical disorders. Journal of Pediatric Psychology, 17, 133-158.

Lewis, M. \& Volkmar, F. (1993). Aspectos clínicos do desenvolvimento na infância e adolescência. Porto Alegre: Artes Médicas.

Mavaux, P. (1981) Hypothyroidism. Em C. D. Brook (Org.), Clinical pediatric endocrinology (pp. 329-339). London: Blackwell.

Neto, E. C. \& Schulte, J. (1998). Rapid and automated essay for thyrotropin in Guthrie cards on the ACS: 180. Clinical Chemistry, 44(11), 2372-2373.

Nolan, T. \& Pless, B. (1986). Emotional correlates and consequences of birth defects. The Journal of Pediatrics, 109(1), 201-216.

Schermann, L., Hagekull, B., Bohlin, G., Person, K. \& Sedin, G. (1997). Interaction between mothers and infants born at risk during the first six months of corrected age. Acta Paediatrica, 86, 864-872.
Stern, D. (1997). A constelaşão da maternidade: O panorama da psicoterapia pais/ bebê. Porto Alegre: Artes Médicas.

Weiland, S. K., Pless, I. B. \& Roghmann, K. J. (1992). Chronic i llness and mental health problems in pediatric practice: results from a survey of primary care previders. Pediatrics, 89, 445-449.

Wendland-Carro, J. \& Piccinini, C. A. (1995). Interação precoce mãe-bebê: perspectivas de intervenção para a promoção do desenvolvimento infantil. Psicologia Reflexão e Crítica, 8, 111-144.

Wolke, D. (1986). Play observation a scheme and emotion ratings (POSER). Manuscrito não-publicado. University Children's Hospital, Munich, West Germany.

Sobre as autoras:

Carla Krás Borges Figueiredo é Psicóloga, Mestre em Psicologia Clínica pela PUC-RS, Professora dos cursos de Psicologia e Pedagogia da Universidade Regional Integrada do Alto Uruguai e das Missões.

Ligia Schermann é Psicóloga, Doutora em Psicologia pela Pontifícia Universidade Católica de São Paulo, Professora do Programa de Pós-Graduação em Saúde Coletiva da Universidade Luterana do Brasil, campus Canoas.

Recebido: 28/07/2000

Revisado: 05/03/2001

Aceite Final: 05/03/2001 


\section{Anexo A}

\section{Protocolo de Observação da Interação Mãe-Criança na Situação de Jogo*}

Nome da criança:

Idade: Sexo: ( ) feminino ( )masculino

Observador:

Itens Maternos

Quantidade de verbalizações

Controle verbal do comportamento

Controle e tutoramento

Sensitividade

Freqüência da expressão emocional positiva

Freqüência da expressão emocional negativa

Itens da Criança

Quantidade de Verbalizações

Orientação da Tarefa

Atividade

Intensidade

Persistência/atenção

Tom emocional

Itens da Díade

Reciprocidade

Controle da sessão

Atmosfera geral

$\begin{array}{lllllll}\text { Nenhuma } & 1 & 2 & 3 & 4 & 5 & \text { Muitas } \\ \text { Muito alto } & 1 & 2 & 3 & 4 & 5 & \text { Muito baixo } \\ \text { Muito freqüente } & 1 & 2 & 3 & 4 & 5 & \text { Nunca } \\ \text { Muito Insensitiva } & 1 & 2 & 3 & 4 & 5 & \text { Muito Sensitiva } \\ \text { Nenhuma } & 1 & 2 & 3 & 4 & 5 & \text { Muita } \\ \text { Nenhuma } & 1 & 2 & 3 & 4 & 5 & \text { Muita }\end{array}$

Nenhuma $\quad 122345$ Muita

Muito baixa $\quad 122345$ Muito alta

Muito calmo $\quad 122345$ Muito ativo

Muito quieto

$\begin{array}{llllll}1 & 2 & 3 & 4 & 5 & \text { Muito intenso }\end{array}$

Muito baixa

$\begin{array}{llllll}1 & 2 & 3 & 4 & 5 & \text { Muito alta }\end{array}$

Muito infeliz

$\begin{array}{lllllll}\text { Nenhuma } & 1 & 2 & 3 & 4 & 5 & \text { Muito } \\ \text { Criança no controle } & 1 & 2 & 3 & 4 & 5 & \text { Mãe no controle } \\ \text { Muito Desacordo } & 1 & 2 & 3 & 4 & 5 & \text { Muita Harmonia }\end{array}$

\footnotetext{
${ }^{*}$ Requisição da descrição das pontuações de cada item do protocolo deve ser encaminhada à segunda autora
} 